\title{
TIMI Coronary Thrombus Grade 1
}

National Cancer Institute

\section{Source}

National Cancer Institute. TIMI Coronary Thrombus Grade 1. NCI Thesaurus. Code C119583.

Possible thrombus present - ang iography demonstrates characteristics such as reduced contrast density, haziness, irregular lesion contour or a smooth convex 'meniscus' at the site of total occlusion suggestive but not diagnostic of thrombus. (Gibson, C. M., de Lemos, J. A., Murphy, S. A., Marble, S. J., McCabe, C. H., Cannon, C. P., Antman, E. M., Braunwald, E. Combination therapy with abciximab reduces angiog raphically evident thrombus in acute myocardial infarction: a TIMI 14 substudy Circulation.

2001;103(21):2550-4.) 